\title{
Geometrical Nonlinear Analysis of Slender Micro Arch-Beams Pulled by Magnetic Loadings
}

\author{
J.D. Yau \\ Department of Architecture \\ Tamkang University, New Taipei City 25137, TAIWAN \\ S.R. Kuo \\ Dept. of Harbor and River Engineering, \\ National Taiwan Ocean University, Keelung, TAIWAN
}

\begin{abstract}
In this study, a nonlinear model of micro arch-beams under the action of electrostatic loadings with an initial air gap is presented. Based on the well-developed 3D beam theory proposed by Yang and Kuo [4], the arch beam is modelled as a series of uniform straight beam elements and the pull-in magnetic force is simulated as an electric-induced force by an electrode lying. Then the nonlinear structural analysis of snap-through and pull-in stability for a simply supported shallow arch subjected to a concentrated electrostatic loading will be investigated. To tackle the path-dependent features of electric forces due to presence of air gaps and nonlinear deformations, this study regards the electric forces as pseudo-forces acting at the arch-beam and solves the structure equations using an incremental-iterative procedure. From the numerical results, the present approach demonstrates that the micro-arch beam has the capacity to withstand further electrostatic loading after the snapthrough jump.
\end{abstract}

Key-Words: - Geometric nonlinear analysis, Micro Arch-Beam, Pull-in stability,

Received: August 29, 2019. Revised: January 24, 2020. Accepted: February 10, 2020.

Published: February 18, 2020.

\section{Introduction}

With the advances of microelectromechanical systems (MEMS) technology in recent years, the applications of MEMS to a variety of micro-sensors and micro-actuators create complex machines with micrometer feature sizes, such as semi-conductor and micro mechanical electric devices [1-3]. For this reason, the geometrical nonlinear phenomena of micro-beams under electrostatic loadings become more pronounced, especially for the pull-in stability and post-buckling of a microbeam subjected to charged substrate. In this study, the pull-in stability of a micro-arch beam subject to a concentrated electro-static loading will be investigated. Based on the welldeveloped 3D beam theory proposed by Yang and Kuo [4], the arch beam can be modelled as a series of uniform straight beam elements and then the snap-through and pull-in stability of the various arch beams under electrostatic loadings are demonstrated in the numerical studies.

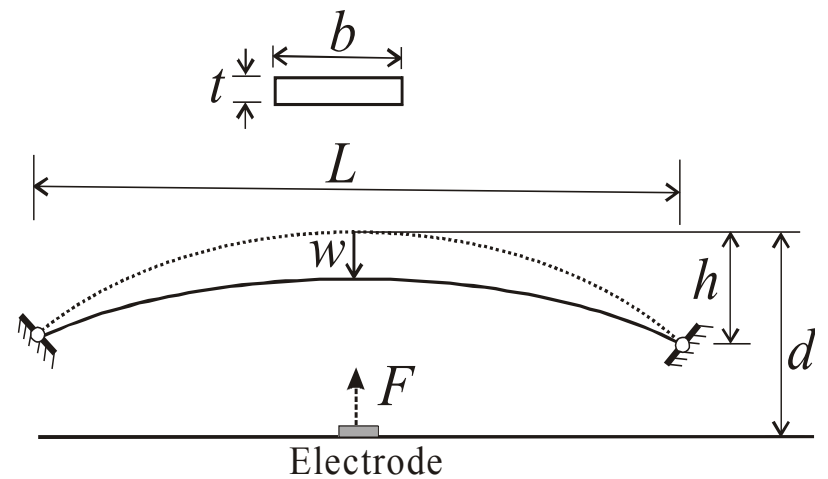

Fig. 1. A micro-arch subject to a concentrated electric force

\section{Problem Formulation}

Figure 1 shows a micro-arch beam under the action of a concentrated electro-static loading, which is induced by an electrode lying under the arch-beam with a generalized electric force $F$ as [1-3]

$$
F=\frac{e \times b \ell}{2}\left(\frac{V}{d-w}\right)^{2}
$$


where $e=$ the dielectric constant of air, $b=$ width, $t=$ thickness, $\ell=$ effective length of electric filed, $d=$ the gap distance, and $V=$ polarization voltage. For the purpose of illustration using finite element approach, the arch beam is modeled as a series of uniform straight beam elements based on the welldeveloped 3D beam theory proposed by Yang and Kuo [4].

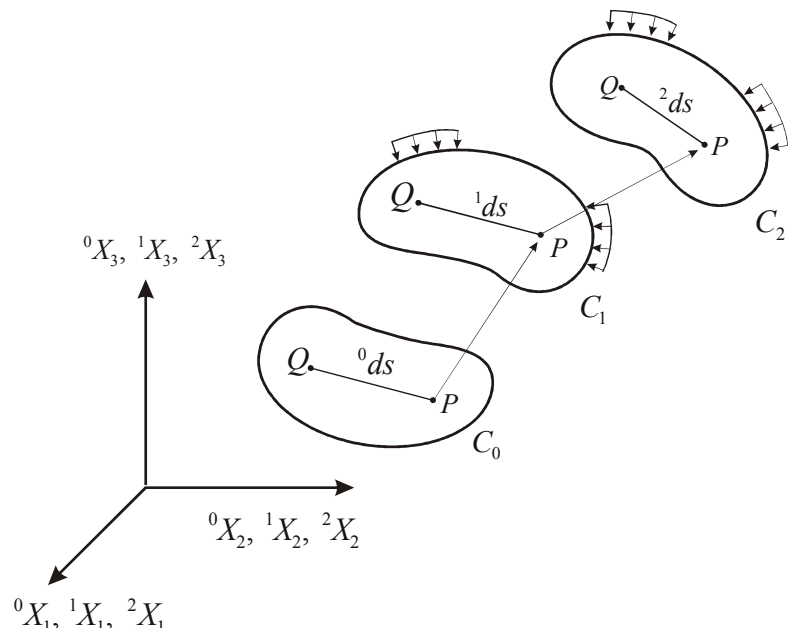

Fig. 2. Motion of body in 3D space.

In an incremental nonlinear analysis based on the updated Lagrangian formulation, as shown in Fig. 2, the incremental element stiffness equation for a planar beam element from the last calculated configuration $C_{1}$ to the current deformed configuration $C_{2}$ can be written as

$\left(\left[k_{e}\right]+\left[k_{g}\right]\right)\{u\}+\left\{{ }^{1} F\right\}=\left\{{ }^{2} F\right\}$

where $\left[k_{e}\right]=$ elastic stiffness matrix, $\left[k_{g}\right]=$ geometric stiffness matrix, $\left\{{ }^{1} F\right\}=$ initial force vector acting at the $C_{1}$ configuration, and $\left\{{ }^{2} F\right\}$ $=$ current force vector at the deformed configuration $C_{2}$. The nodal displacement vector $\{u\}$ for the incremental step from $C_{1}$ to $C_{2}$ in terms of the $x^{1}-y^{1}$ coordinates is

$\{u\}=\left\langle\begin{array}{llllll}u_{a} & v_{a} & \theta_{a} & u_{b} & v_{b} & \theta_{b}\end{array}\right\rangle$.

Correspondingly, the initial force vector $\left\{{ }^{1} F\right\}$ acting on the element is

$\left\{{ }^{1} F\right\}=\left\langle\begin{array}{llllll}F_{x a} & F_{z a} & M_{y a} & F_{x b} & F_{z b} & M_{y b}\end{array}\right\rangle^{T}$

By equilibrium,

$\left\{{ }^{1} F\right\}=\left\langle\begin{array}{llllll}-F_{x b} & -F_{z b} & M_{y a} & F_{x b} & F_{z b} & M_{y b}\end{array}\right\rangle^{T}$

where $F_{x b}=$ axial force, $F_{z b}=$ shear force, $M_{y b}=$ bending moment, and $F_{z b}=\left(M_{y a}+M_{y b}\right) / L$.

Before we proceed with derivation of the geometric stiffness matrix for the curved beam element, two important concepts must be mentioned here. First, as far as the rigid behavior is concerned, the behavior of a twonode curved beam depends exclusively on the behavior of the two end points $a$ and $b$, but not on the shape or elastic properties of the element, and thus is identical to the behavior of the straight beam consisting of the same end points $a$ and $b$. Second, the geometric stiffness matrix for a two-dimensional straight beam can be derived in an approximate, but rather accurate manner, by considering only the rigid body rotations [4], due to the fact that that the rigid body displacements constitute a great portion of the buckling displacement of the beam elements. In this paper, the geometric stiffness matrix of a two-dimensional straight beam element based on the rigid concept will be first summarized.

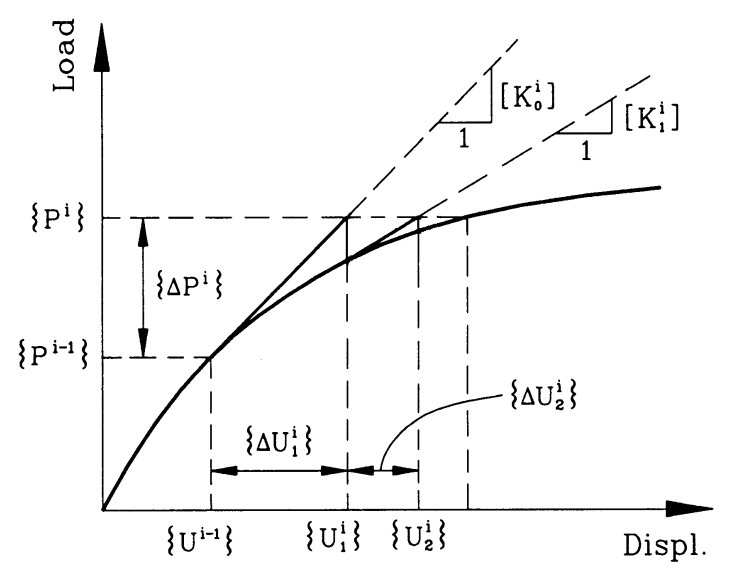

Fig. 3 Predictor phase of nonlinear analysis

On the other hand, the electric force shown in Eq. (1) features the characteristics of pathdependent due to presence of air gaps and nonlinear deformations, from which the geometric stiffness matrix of a beam element would become elaborate in finite element formulation stage. To tackle this problem, this study will regard the electric forces as pseudoforces acting at the arch-beam and then solve the structure equations using an incrementaliterative procedure based on the concept of predictor, corrector and equilibrium checking phases, as plotted in Fig. 3. They are summarized as follows: 
(1) Predictor phase

To solve the structural displacement increments under given load increments from the structural equations. It is noted that the geometric stiffness matrices with nonlinear deformation for electric forces will be skipped in programming at this stage.

\section{(2) Corrector phase}

To recover the element forces at deformed configuration from the structural deformations via the predictor phase (see Fig. 4)

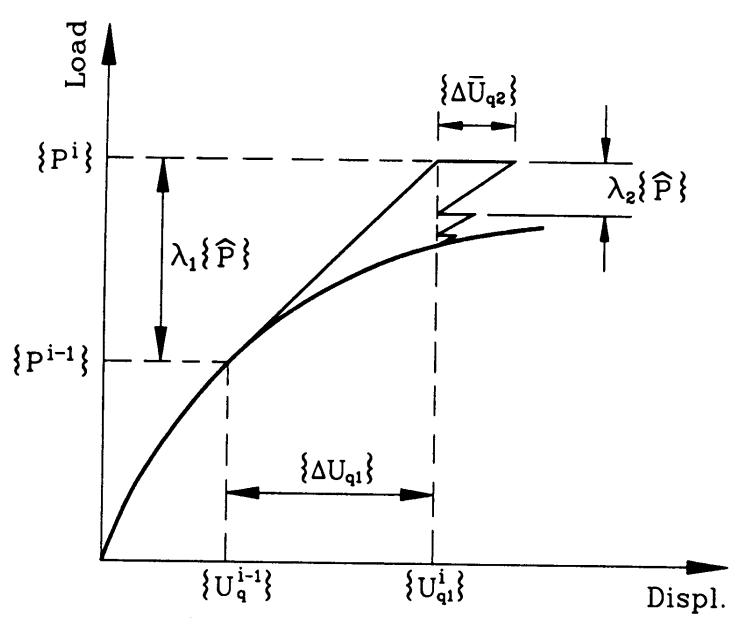

Fig. 4 Corrector phase of nonlinear analysis

(3) Equilibrium-checking phase

To update the geometry and confirm the acting point of the concentrated electro-static force and compare the internal forces computed in the deformed configuration of the micro-arch with the total applied loads to obtain the unbalanced forces of the structure. It is noted that the deformation-dependent nature of electric forces has been taken into account in this phase.

(4) Check the accuracy of solution for next iterations or incremental steps.

\section{Geometrical nonlinear analysis based on incremental-iterative procedure}

To solve the pull-in stability of a micro-arch beam under the action of a concentrated electrostatic force with a gap distance, an incrementaliterative procedure is performed for geometric nonlinear analysis. Three key phases are essential to an incremental-iterative nonlinear analysis, i.e., the predictor, corrector and equilibrium checking phases [5]. The predictor phase is concerned with solution of the structural displacement increments under given load increments from the structural equations, which may affect the speed of convergence or the number of iterations. The corrector phase deals with recovery of the element forces at deformed configuration from the existing nodal forces and the element displacement increments made available from the structural displacement increments via the predictor phase. This phase determines primarily the accuracy of the solution. After all the element forces are computed and expressed in the deformed configuration of the structure, one can compare them with the total applied loads to obtain the unbalanced forces of the structure at deformed configuration, as is typical for the equilibriumchecking phase. It is emphasized that the deformation-dependent effect of electric forces will be taken into account in this phase so that the geometric stiffness matrices with nonlinear deformations can be skipped in programming. Whenever the unbalanced forces are greater than preset tolerances, the unbalanced forces should be regarded as the applied loads and another iteration involving the three phases should be repeated.

For the purpose of tracing the load-deflection response of a curved beam structure under the action of magnetic forces, the generalized displacement control (GDC) method proposed by Yang and Shieh [6] will be adopted for its general stability in dealing with multi loops in the post-buckling response. By this method, the load increment size is determined as a function of the general stiffness parameter (GSP) [6], which serves as a reliable indicator for reversing the direction of loading once a limit point is detected.

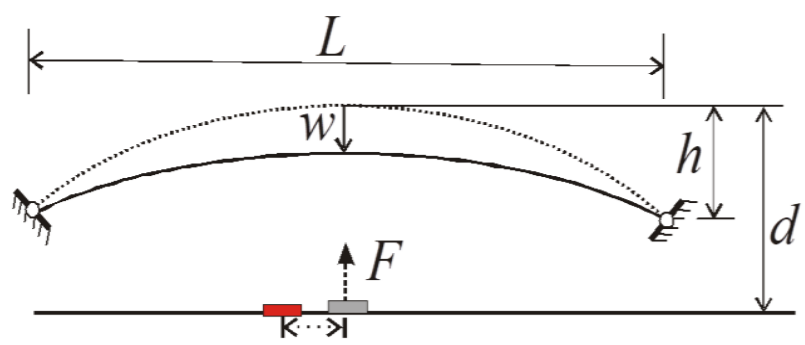

Electrode

Fig. 5 A simply supported micro-arch subject to an electric force with perfect/imperfect loading 


\section{Illustrated example}

The micro-arch beam depicted in Fig. 5 is a shallow arch and made of silicon with Young's modulus $E=160 \mathrm{Gpa}$ and Poisson ratio $v=$ 0.27 . The properties of the arch-beam computed here are given as: $b=9 \mu \mathrm{m}, t=2.2 \mu \mathrm{m}, e=$ $8.854 \times 10^{-12} \mathrm{~F} / \mathrm{m}, d=20 \mu \mathrm{m}, h=5 \mu \mathrm{m}, L=$ $500 \mu \mathrm{m}$. The effective length of the electrode $\ell$ is $1 \mu \mathrm{m}$. Two quasi-electrostatic loading cases are considered, one is that the stationary electrode is exactly located at the mid-span (perfect electrostatic loading) and the second located at the left side of $10 \mu \mathrm{m}$ from the midspan ( for imperfect electrostatic loading).

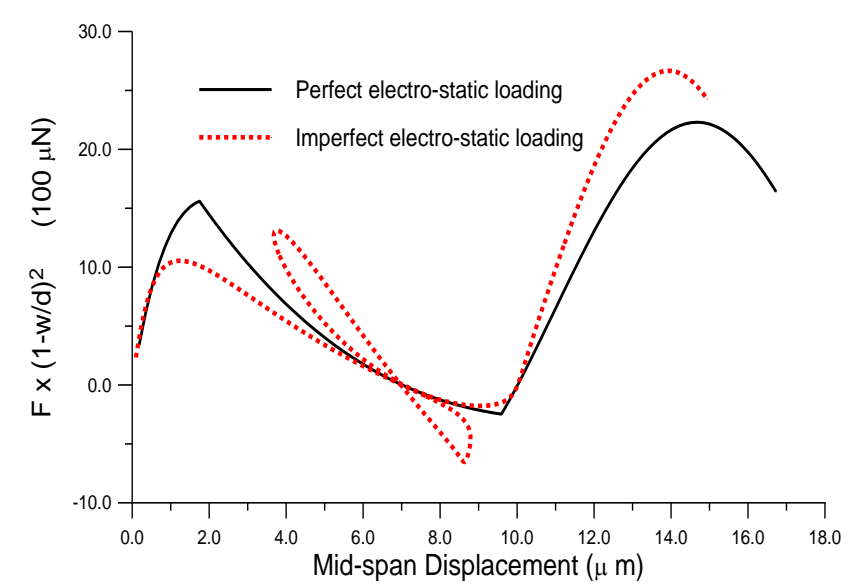

Fig. 6. Load-deflection curves for the microarch beam under electro-loadings.

The geometrical nonlinear analysis procedure is carried out until the deformed arch-beam is quite closed to the surface of stationary electrode. The numerical results of midpoint displacement and electric force have been plotted in Fig. 6. The post-buckling phenomena of pull-in stability occur around the mid-span vertical displacement $2 \mu \mathrm{m}$. Moreover, after the snap-through jump, the present approach demonstrates that the micro-arch has the capacity to withstand further electrostatic loading.

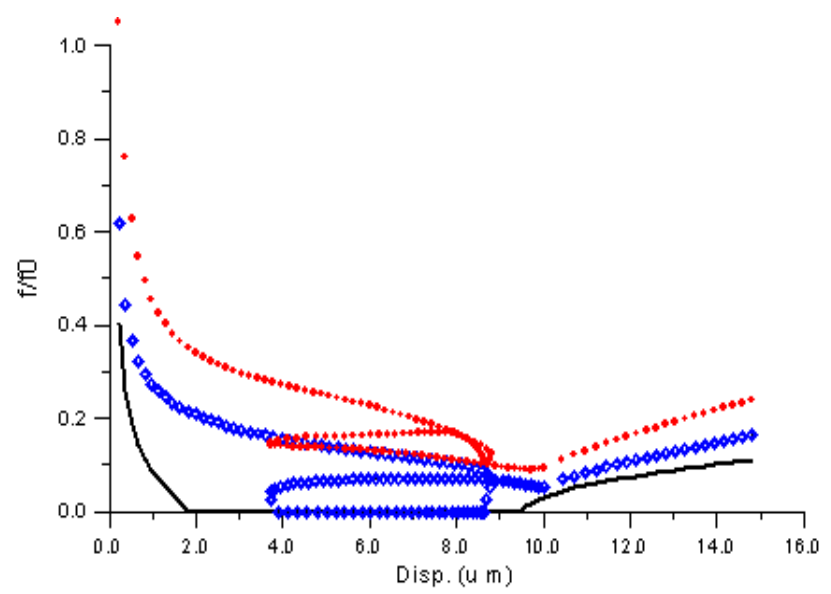

Fig. 7 Relationship between the frequency and the displacement of the micro-arch

Moreover, Fig. 7 shows the relationship between the frequency and the displacement of the micro-arch under the action of a concentrated electric force. The present approach gives an efficient algorithm to detect the change of structural stiffness.

\section{Concluding remarks}

Nonlinear deformation analysis and eigenvalue solutions would consume much CPU time in numerical computations, especially for the computational problem by using an efficient algorithm to detect the change of structural stiffness.

To circumvent the mathematical hindrance encountered in formulation of the electric force acting on a arch-beam by the conventional finite element approach, this study regards the electric forces as pseudo-forces acting at the arch-beam and then solve the structure equations using an incremental-iterative procedure. The advantage of the present approach is that there is no need to deal with the path-dependent force due to presence of air gaps and nonlinear deformations. From the numerical studies, the present approach demonstrates that the micro-arch beam has the capacity to withstand further electrostatic loading after the snap-through jump.

\section{Acknowledgement}

This work is supported by the Ministry of Science \& Technology of Taiwan via the grant No. (MOST 106-2923-E-002-007-MY3, 1072221-E-032-002-MY2). The first author would 
like to thank the financial support of Tamkang University for him to present his recent research work.

\section{References:}

1. Abdel-Rahman, E.M., Younis M.I., Nayfeh A.H., Characterization of the mechanical behavior of an electrically actuated microbeam, J. Micromech. Microeng., 2002; 12, 759-766.

2. Collenz, A., De Bona, F., Gugliotta, A., Som `a, A., Large deflections of microbeams under electrostatic loads, J. Micromech. Microeng., 2004; 14, 365-373.

3. Zhang Y., Wang Y., Li Z., Huang Y., Li D., Snap-Through and Pull-In Instabilities of an Arch-Shaped Beam Under an Electrostatic Loading, J. Microelectromechical Systems, 2007; 16(3), 684-693.

4. Yang Y.B., Kuo S.R., Theory and Analysis of Nonlinear Framed Structures, Singapore: Prentice Hall; 1994.

5. Yang, Y.B., Lin, S.P., and Leu, L.J., Solution Strategy and Rigid Element for Nonlinear Analysis of Elastically Structures Based on Updated Lagrangian Formulation. Eng. Struct. 2007; 29(6), 1189-1200.

6. Yang Y.B., Shieh M.S., Solution method for nonlinear problems with multiple critical points, AIAA J. 1990; 28(12):2110-2116. 